\title{
Is Ginger Effective in Reducing Post-tonsillectomy Morbidity? A Prospective Randomised Clinical Trial
}

\author{
İlker Koçak ${ }^{1}$ Cemil Yücepur² • Ozan Gökler ${ }^{1}$ \\ ${ }^{1}$ Department of Otolaryngology, Koç University Hospital, Istanbul; ${ }^{2}$ Department of Otolaryngology, Darica Farabi State Hospital, Kocaeli, Turkey
}

Objectives. An assessment of the effects of ginger on pain, nausea, vomiting, bleeding, and wound site healing that occur after tonsillectomy.

Methods. This prospective clinical study was participated by 49 patients aged 18-45 years out of 56 patients that underwent tonsillectomy and regularly attended follow-up visits. The patients were randomly divided into two groups. Group 1 consisted of 23 patients that used ginger capsules and group 2 consisted of 26 patients that did not use ginger capsules in addition to the routine antibiotic and paracetamol treatment following tonsillectomy. The pain, nausea, vomiting, and bleeding scores of patients were assessed on days 1, 4, 7, and 10 by using the visual analogue scale. Similarly, the epithelialization degrees of tonsillar bed were assessed on postoperative days 1, 4, 7, and 10 and regular oral intake times of patients were recorded.

Results. There were no differences between groups in terms of age and sex. On the postoperative days 1, 4, 7, and 10, the pain scores were lower in the group 1 (all $P<0.001$ ) and there were no differences between the two groups in terms of postoperative nausea, vomiting, and bleeding scores $(P=0.35$ and $P=0.53)$. On the postoperative days 7 and 10 , epithelialization in the tonsil bed was quicker in the group $1(P=0.041$ and $P=0.026)$ and the regular oral intake time was earlier in group $1(P<0.001)$.

Conclusion. In this study, ginger relieved pain, accelerated wound site epithelialization, and decreased duration of oral intake following tonsillectomy. It did not result in postoperative bleeding or any other complications. Ginger can be recommended as an adjunct to routine medical treatment for decreasing morbidity following tonsillectomy.

Keywords. Ginger; Tonsillectomy; Pain; Wound Healing

\section{INTRODUCTION}

Tonsillectomy is globally one of the most frequently performed surgeries on children and adults. Even though we currently have advanced surgical techniques, patients often complain about postoperative nausea, vomiting, throat ache, and consequent decrease in oral intake. The reasons for this pain include secondary healing of the wound site, inflammation, pharyngeal muscle spasm and glossopharyngeus and vagus nerve irritation. The pain persists until approximately $2-3$ weeks as the pharyngeal

- Received April 1, 2017

Revised June 24, 2017

Accepted July 28, 2017

- Corresponding author: İlker Koçak

Department of Otolaryngology, Koç University Hospital, Davutpasa Cad.

No. 4,Topkapi 34010, Istanbul, Turkey

Tel: +90-555-711-6870, Fax: +90-212-311-3410

E-mail: ikocak@kuh.ku.edu.tr muscles are covered by the mucosa. In order to relieve postoperative pain following tonsillectomy, several methods and medical therapeutic agents have been used such as hyaluronic acid [1], magnesium [2], opioid [3], honey intake [4], sucralfate in the tonsil region [5], and autologous serum [6].

Ginger, which is scientifically known as Zingiber officinale, is a plant that has anti-inflammatory and analgesic properties known in many Asian countries [7]. The anti-inflammatory property of ginger originates from compounds such as gingerols, shogaols, and paradols in its composition. Studies performed have also shown that ginger not only brings about lipoxygenase inhibition, thereby reducing the synthesis of inflammatory leukotrienes [8], but it also shows an anti-inflammatory effect by enabling cytokine inhibition in a way similar to betamethasone [9].

Additionally, studies conducted have also shown that ginger is effective in enhancing wound healing $[10,11]$, preventing postoperative nausea and vomiting (antiemetic) [12], relieving pain

Copyright @ 2018 by Korean Society of Otorhinolaryngology-Head and Neck Surgery.

This is an open-access article distributed under the terms of the Creative Commons Attribution Non-Commercial License (http://creativecommons.org/licenses/by-nc/4.0)

which permits unrestricted non-commercial use, distribution, and reproduction in any medium, provided the original work is properly cited. 
due to dysmenorrhea [13-15], osteoarthritis [16], and rheumatoid arthritis [17]. As can be seen, there are several studies where ginger has been clinically applied, however, our literature review did not show any studies investigating the intake of ginger for pain and morbidity that develop after tonsillectomy. The aim of this study is to investigate the effects of ginger on pain following tonsillectomy and to ensure that patients begin oral intake in a shorter period of time following the operation.

\section{MATERIALS AND METHODS}

The study was initiated after the approval was obtained from the Kocaeli University Clinical Research Ethics Committee (approval No. 4/15). All procedures performed in studies involving human participants were in accordance with the ethical standards of the institutional and/or national research committee and with the Helsinki declaration and its later amendments or comparable ethical standards. Our study included 56 patients aged 18-45 years, who received the indication for tonsillectomy due to recurrent tonsillitis, snoring due to tonsillar hypertrophy or chronic tonsillitis resistant to medical treatment between September 2015 and February 2017. Patients who previously underwent tonsillotomy, underwent another surgery simultaneously with tonsillectomy (such as uvulopalatopharyngoplasty or other snoring surgeries), had mental retardation, had to continuously use painkillers, and had known allergy to ginger were excluded from the study. Written and oral informed consents were obtained from the patients prior to the study. The patients were randomly divided into two groups by a department nurse on the day of surgery.Tonsillectomy was performed by a surgeon under general anesthesia using the conventional cold dissection technique. Bleeding control was done using a bipolar cautery. Patients in group $1(n=28)$ were administered amoxicillin/clavulanate $(1,000$ $\mathrm{mg}$ ) two times per day, paracetamol tablets $(500 \mathrm{mg})$ three times per day, and ginger capsules (500 mg; Ginger Root, Solgar, Leonia, NJ, USA) two times per day for 7 days postoperatively. Patients in group $2(\mathrm{n}=28)$ were administered amoxicillin/clavulanate $(1,000 \mathrm{mg})$ two times per day, paracetamol tablets $(500 \mathrm{mg})$ three times per day for 7 days postoperatively. The patients did not know that the ginger capsules had analgesic properties. The patient's pain was evaluated on days $1,4,7$, and 10 by an otorhi-

\section{H I G G H L L I}

- Ginger relieved pain, accelerated wound site epithelialization, and decreased duration of oral intake following tonsillectomy.

- Ginger did not result in postoperative bleeding or any other complications.

- Ginger can be recommended as an adjunct to routine medical treatment for decreasing morbidity following tonsillectomy.
Table 1. Bleeding and nausea/vomiting scoring

\begin{tabular}{lc}
\hline Variable & Score \\
\hline Bleeding score & \\
None & 0 \\
Minimal & 1 \\
Moderate & 2 \\
Severe & 3 \\
Nausea/vomiting score & \\
None & 0 \\
Nausea & 1 \\
Dry heaves & 2 \\
Vomiting & 3 \\
\hline
\end{tabular}

Table 2. The degree of epithelialization of tonsiller bed

\begin{tabular}{ll}
\hline Grade & \multicolumn{1}{c}{ Degree of epithelialization } \\
\hline 1 & Complete fibrin coverage of tonsillar bed \\
2 & Initiation of epithelialization (epithelia covering less than 30\%) \\
3 & Epithelialized by half (epithelia covering 30\%-75\%) \\
4 & Near complete epithelialization (epithelia covering more than $75 \%$ ) \\
5 & Complete epithelialization of tonsillar bed \\
\hline
\end{tabular}

nolaryngologist who was unaware of the procedure on the basis of visual analogue scale ( 0 , no pain; 10 , severe pain).

Postoperative nausea, vomiting, and bleeding complaints were evaluated by an otorhinolaryngologist who was unaware of the procedure on days $1,4,7$, and 10 on the basis of the scale in Table 1 [2]. Epithelialization in the tonsillar bed was also evaluated by an otorhinolaryngologist who was unaware of the procedure on days $1,4,7$, and 10 using the method previously defined in the literature (Table 2) [4]. The patients were recommended to have a soft diet in the first week. Furthermore, they were asked on which postoperative day they began regular oral intake.

\section{Statistical analysis}

Statistical analysis was carried out using IBM SPSS ver. 22.0 (IBM Corp., Armonk, NY, USA). All the quantitative variables were estimated using measures of central location (i.e., mean and median) and measures of dispersion (i.e., standard deviation). Data normality was checked using the KolmogorovSmirnov tests of normality.

Mann-Whitney $U$-test and independent $t$-test were used in between-group evaluation for quantity data (age, pain scores, bleeding scores, nausea/vomiting scores, time to regular oral intake).

Chi-square test was used for the comparison of qualitative data (gender, degree of epithelialization). A $P$-value $<0.05$ was considered statistically significant.

\section{RESULTS}

The study included 56 patients ( 31 males and 25 females, aged 18 to 45 years) who underwent tonsillectomy surgery. Out of 
these patients, seven patients (five patients in group 1 and two patients in group 2) did not regularly attend their follow-up visits. Group $1(n=23)$ had 10 male and 13 female patients between 21 and 45 years (average, $29.3 \pm 7$ years) of age. Group $2(n=26)$ had 15 male and 11 female patients between 18 and 39 years (average, $27.1 \pm 8$ years) of age. There were no statistically significant differences between two groups in terms of gender, age, body mass index, and indication for tonsillectomy (Table 3).

When the groups were compared in terms of pain score, it was seen that the pain score of the group that took ginger (group 1) was lower than that of the group 2 on postoperative on days 1, 4, 7, and 10 (all $P<0.001$ ) (Fig. 1). This is an indication that patients who take ginger following tonsillectomy have less pain

Table 3. Patients' baseline characteristics

\begin{tabular}{lccc}
\hline Characteristic & $\begin{array}{c}\text { Group 1 } \\
\text { (ginger) }\end{array}$ & $\begin{array}{c}\text { Group 2 } \\
\text { (control) }\end{array}$ & $P$-value \\
\hline Age $(\mathrm{yr})$ & $29.3 \pm 7$ & $27.1 \pm 8$ & 0.335 \\
Sex & & & 0.396 \\
$\quad$ Female & 13 & 11 & \\
$\quad$ Male & 10 & 15 & \\
Body mass index $\left(\mathrm{kg} / \mathrm{m}^{2}\right)$ & $25.2 \pm 4.0$ & $27.1 \pm 4.6$ & 0.143 \\
Indications for tonsillectomy & & & 0.292 \\
$\quad$ Chronic tonsillitis & 8 & 14 & \\
$\quad$ Recurrent tonsillitis & 11 & 7 & \\
$\quad$ Tonsillar hypertrophy & 4 & 5 & \\
\hline
\end{tabular}

Values are presented as mean \pm standard deviation.

a)Difference is significant at the significance level $P<0.05$.

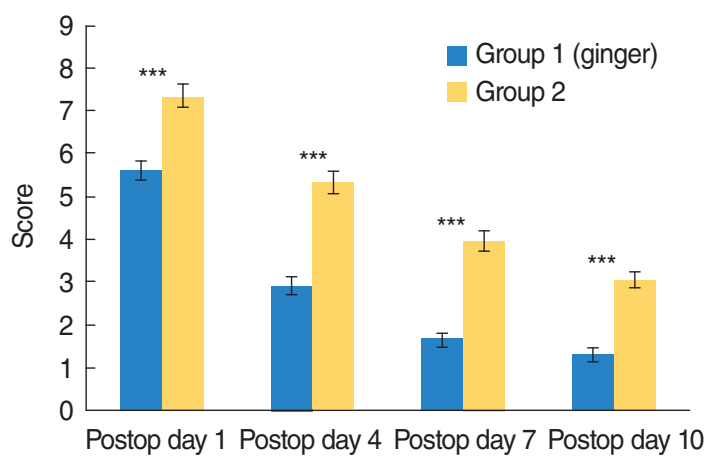

Fig. 1. Comparison of pain visual analog scale scores on postoperative (postop) days 1, 4, 7, and 10. Vertical error bars represent standard error. ${ }^{\star \star \star} P<0.001$. during the postoperative period.

Even though bleeding was observed in two patients in group 2 on postoperative days 3 and 8 , there are no statistical differences between the two groups in terms of bleeding scores $(P>0.05)$. As can be seen here, ginger did not negatively influence bleeding. Additionally, no significant differences were seen between the two groups on postoperative days $1,4,7$, and, 10 with respect to the vomiting and nausea scores $(P>0.05)$.

When the degree of epithelialization in the tonsillar bed was compared, there were no differences between the two groups on postoperative days 1 and 4 (all $P>0.05$ ) while it was seen that epithelialization was better in the group 1 on postoperative days 7 and $10(P=0.041$ and $P=0.026)$ (Fig. 2, Table 4). Seventy-four percent of patients in group 1 and $42 \%$ of patients in group 2 showed grade 4 epithelialization on day 10. Judging from this result, it could be suggested that ginger accelerates wound healing in line with the literature data $[10,11]$.

The regular oral intake time of the patients in group 1 was postoperatively $3.2 \pm 0.6$ days, while this was observed to be $4.6 \pm 0.9$ days for group 2 . The patients who took ginger began oral intake at an earlier stage $(P<0.001)$.

\section{DISCUSSION}

Throat ache is a complaint that frequently develops following tonsillectomy surgery and it may lead to undesired situations such as prolonged hospitalization, poor oral intake, dehydration, and late return to daily life. In the past, several agents were used to relieve pain following tonsillectomy [1-6] and they each have
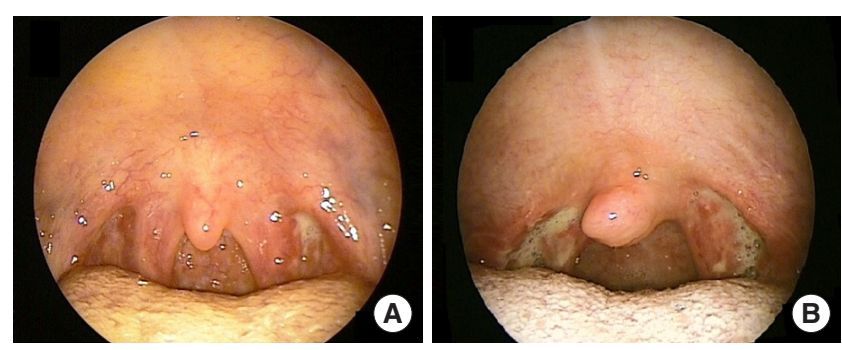

Fig. 2. Representative view of tonsillar bed at postoperative day 10. (A) Patient who took ginger, (group 1), (B) patient who did not take ginger (group 2).

Table 4. Comparison of epithelialization degrees on postop days 4, 7, and 10

\begin{tabular}{|c|c|c|c|c|c|c|c|}
\hline \multirow{2}{*}{ Epithelialization degree } & \multicolumn{2}{|c|}{ Postoperative day 4} & \multicolumn{3}{|c|}{ Postoperative day 7} & \multicolumn{2}{|c|}{ Postoperative day 10} \\
\hline & Grade 1 & Grade 2 & Grade 2 & Grade 3 & Grade 4 & Grade 3 & Grade 4 \\
\hline Group 1 (ginger) & $5(22)$ & $18(78)$ & $4(17)$ & $18(79)$ & $1(4)$ & $6(26)$ & $17(74)$ \\
\hline Group 2 (control) & $12(46)$ & $14(54)$ & $11(42)$ & $15(58)$ & 0 & $15(58)$ & $11(42)$ \\
\hline$P$-value ${ }^{\text {a) }}$ & \multicolumn{2}{|c|}{0.073} & \multicolumn{3}{|c|}{0.041} & \multicolumn{2}{|c|}{0.026} \\
\hline
\end{tabular}

Values are presented as number (\%).

a) Chi-square test. 
individual advantages and disadvantages. For that reason, there is currently not a consensus on relieving pain after tonsillectomy. Opioids and nonsteroidal anti-inflammatory drugs (NSAIDs) are the drugs that are most frequently used to relieve pain after tonsillectomy. As it is well-known, opioids may lead to respiratory depression and sedation. As for NSAIDs, they are commonly used even though their use is still controversial since they have effects on platelets and prolong bleeding time [18]. Paracetamol is a reliable drug to be taken after tonsillectomy; however, it is not adequately effective against throat ache and its use at a high dose may result in hepatotoxicity [19].

Ginger, which is more often used globally to add flavor to dishes is a plant known in Asian countries with its anti-inflammatory and analgesic properties. Shirvani et al. [13] and Rahnama et al. [14] showed that ginger reduced pain related to dysmenorrhea. Ozgoli et al. [15] reported that ginger was as effective as mefenamic acid and ibuprofen as a painkiller. Bartels et al. [16] showed that ginger was effective in treating osteoarthritis-related pain and Al-Nahain et al. [17] showed that it was effective in symptomatic treatment of pain associated with rheumatoid arthritis. Similarly, it was stated in the literature that ginger was effective against throat ache [20]. Ginger enables cyclooxygenase and lipoxygenase enzyme inhibition through the arachidonic acid metabolism. Consequently, it shows analgesic and anti-inflammatory effects by ensuring prostaglandin and leukotriene suppression [14]. In another mechanism, it shows an antiinflammatory effect via cytokine inhibition in a way similar to betamethasone [9]. In conclusion, it has been used to control pain in several diseases as seen. However, we have not found any studies in the literature that investigated the efficacy of ginger in relieving pain following tonsillectomy. In the line of these studies, we considered that ginger could relieve postoperative pain following tonsillectomy. In the study that we conducted, we observed that patients who took ginger after tonsillectomy had less pain on postoperative days 1 to 10 as compared to patients who did not take it (postoperative days 1,4,7,10; $P<0.001$ ). Judging by this result, it could be concluded that ginger helps relieving pain that develops after tonsillectomy.

Studies in the literature have shown that ginger is effective against postoperative nausea and vomiting $[12,21]$. The mechanism of action here is not entirely clear. Some authors advocate that it shows an antiemetic effect by reducing stomach contractions and increasing the intestinal system activity [21], while other authors state that it prevents nausea and vomiting by having an inhibitory effect on 5-HT serotonin receptors in a way similar to antiemetic drugs. We also investigated the effect of ginger on nausea and vomiting after tonsillectomy; however, we did not see any differences between the two groups in terms of postoperative nausea and vomiting $(P>0.05)$. We believe that we could not see a significant difference since the patients took ginger after the operation in our study, whereas ginger was given approximately 1 hour before the operation to patients for post- operative antiemetic effect in the studies performed $[12,21]$.

Several studies investigated the effects of ginger on coagulation. Jiang et al. [22] reported that ginger showed an anticoagulant effect in a way similar to aspirin. In another study, it was demonstrated that ginger did not have any effects on platelet aggregation [23]. Additionally, Kashefi et al. [24] showed that ginger reduced menstrual bleeding in a study that they conducted. In our study, none of the patients that took ginger had bleeding. Postoperative bleeding was observed in two patients in the group that did not take ginger, although there were no statistically significant differences between the two groups in terms of postoperative bleeding. Based on this result, it could be suggested that ginger does not have any side effects related to bleeding.

Another property of ginger is that it accelerates wound healing. In two experimental studies, it was demonstrated that ginger contributed to the enhancement of wound healing $[10,11]$. The role of ginger in wound healing has not been entirely understood. It was considered that this could be because it supported blood vessel formation in the healing tissue and increased the level of type 1 collagen [11]. We also saw in our study that the tonsillar bed epithelialization was statistically significantly better in patients that took ginger on postoperative days 7 and 10 as compared to those who did not take it (Fig. 2). We can conclude that ginger accelerates healing in the tonsillar bed.

Delayed begin to oral intake and consequent dehydration are complications that may develop after tonsillectomy. Early begin to oral intake after tonsillectomy is important in the sense that it ensures that patients recover rapidly and shortens the hospital stay. In this study that we conducted, we saw that patients taking ginger began oral intake at an earlier stage $(P<0.001)$. We believe that they started regular oral intake earlier because patients taking ginger have less postoperative pain.

Rahnama et al. [14] reported in their study that some subjects who took ginger had side effects such as heartburn. In the study by Rahnama et al., heartburn may have arisen out of the use of ginger at a high dose. In our study, patients taking ginger did not have any gastrointestinal effects such as heartburn and similar issues. Furthermore, bleeding, which is a potential side effect of ginger, was not seen in patients taking ginger in our study, either.This is probably due to the fact that we used ginger at a lower dose.

The studies have shown that ginger was as effective as ibuprofen (NSAID) the management of pain after dental surgery [25] and in patients with osteoarthritis [26], also as effective as ibuprofen and mefenamic acid in relieving menstrual pain $[13,15]$. Furthermore, ginger has been accepted as safe by both the Food and Drug Administration [7] with no report of severe side effects or drug interactions in Germany's Commission E Monograph [27]. In contrast, adverse effects of NSAIDs include gastrointestinal disorders (such as nausea, dyspepsia, and vomiting) and central nervous system symptoms, nephrotoxic and hepatotoxic effects, hematological abnormalities, and bronchospasm [13]. Sometimes, tonsillectomy is associated with nausea and 
vomiting. Although NSAIDs may lead nausea and vomiting, ginger also alleviates these symptoms $[12,21]$. Ginger may be preferred for patients who do not want to use NSAIDs because of its adverse effects.

Considering cost of ginger capsule, its price is about $\$ 14$ (for 60 capsules). Although the costs of different NSAID agents vary, average price of NSAID drugs are approximately $\$ 10$ (for 20 tablets). Ginger capsules are not more expensive than NSAIDs analgesics. Also, ginger can reduce postoperative pain and consequently the need for extra analgesic consumption. Furthermore, ginger may reduce the treatment costs due to the side effects of NSAIDs (such as bleeding and gastrointestinal problems) or the length of stay in the hospital because of late oral intake. For all these reasons, we think that ginger can be cost-effective for postoperative management. We currently recommend patients undergoing tonsillectomy to take ginger alongside analgesic drugs such as paracetamol for pain control and we receive positive feedback.

One of the limitations of our study is that the sample size is small even though it is statistically adequate. In the future, studies with several patients' groups and multiple centers may be conducted. Another limitation is related to the dose of ginger. There is not an existing consensus on the maximum dose of ginger per day. Even though maximum daily dose is considered to be $2 \mathrm{~g}$ in Europe and America, the daily dose goes up to $9 \mathrm{~g}$ in Asia [28]. We determined the dose of ginger in the study we conducted by referencing the previous studies in the literature. Also, other limitations of the study could be attributed to an absence of placebo or other NSAIDs treatment in the control group. Although our study lacked a placebo group, it was still prospectively randomized controlled study and compared the treatment group with a relevant control group and as such has a low risk of bias. Placebo-controlled or comparative studies are needed to support our findings and minimize bias in data collection.

This study has shown that ginger has facilitated pain management following tonsillectomy. Additionally, patients who took ginger had a faster tonsil bed epithelialization and an earlier begin to regular oral intake. Also, no side effects were observed. For that reason, we recommend patients to take ginger alongside analgesic drugs such as paracetamol following tonsillectomy. However, studies with larger sample sizes and multiple centers are needed to support our results and to demonstrate the efficacy of ginger against post-tonsillectomy morbidities.

\section{CONFLICT OF INTEREST}

No potential conflict of interest relevant to this article was reported.

\section{REFERENCES}

1. Hanci D, Altun H. Effectiveness of hyaluronic acid in post-tonsillectomy pain relief and wound healing: a prospective, double-blind, controlled clinical study. Int J Pediatr Otorhinolaryngol. 2015 Sep; 79(9):1388-92.

2. Tugrul S, Degirmenci N, Eren SB, Dogan R, Veyseller B, Ozturan O. Analgesic effect of magnesium in post-tonsillectomy patients: a prospective randomized clinical trial. Eur Arch Otorhinolaryngol. 2015 Sep;272(9):2483-7.

3. Fukuda K. Intravenous opioid anesthetics. In: Miller RD, editor. Miller's anesthesia. Kidlington (UK): Churchill Livingstone Elsevier; 2005. p. 379-437.

4. Ozlugedik S, Genc S, Unal A, Elhan AH, Tezer M, Titiz A. Can postoperative pains following tonsillectomy be relieved by honey? A prospective, randomized, placebo controlled preliminary study. Int J Pediatr Otorhinolaryngol. 2006 Nov;70(11):1929-34.

5. Siupsinskiene N, Zekoniene J, Padervinskis E, Zekonis G, Vaitkus S. Efficacy of sucralfate for the treatment of post-tonsillectomy symptoms. Eur Arch Otorhinolaryngol. 2015 Feb;272(2):271-8.

6. Kara M, Erdogan H, Altinisik HB, Aylanc H, Guclu O, Derekoy FS. Does topical use of autologous serum help to reduce post-tonsillectomy morbidity? A prospective, controlled preliminary study. J Laryngol Otol. 2016 Jul;130(7):662-8.

7. Ali BH, Blunden G, Tanira MO, Nemmar A. Some phytochemical, pharmacological and toxicological properties of ginger (Zingiber officinale Roscoe): a review of recent research. Food Chem Toxicol. 2008 Feb;46(2):409-20.

8. Kiuchi F, Iwakami S, Shibuya M, Hanaoka F, Sankawa U. Inhibition of prostaglandin and leukotriene biosynthesis by gingerols and diarylheptanoids. Chem Pharm Bull (Tokyo). 1992 Feb;40(2):387-91.

9. Ribel-Madsen S, Bartels EM, Stockmarr A, Borgwardt A, Cornett C, Danneskiold-Samsoe B, et al. A synoviocyte model for osteoarthritis and rheumatoid arthritis: response to ibuprofen, betamethasone, and ginger extract. A cross-sectional in vitro study. Arthritis. 2012; 2012:505842. https://doi.org/10.1155/2012/505842.

10. Bakht MA, Alajmi MF, Alam P, Alam A, Alam P, Aljarba TM. Theoretical and experimental study on lipophilicity and wound healing activity of ginger compounds. Asian Pac J Trop Biomed. 2014 Apr;4 (4):329-33.

11. Bhagavathula N, Warner RL, DaSilva M, McClintock SD, Barron A, Aslam MN, et al. A combination of curcumin and ginger extract improves abrasion wound healing in corticosteroid-impaired hairless rat skin. Wound Repair Regen. 2009 May-Jun;17(3):360-6.

12. Montazeri AS, Hamidzadeh A, Raei M, Mohammadiun M, Montazeri AS, Mirshahi R, et al. Evaluation of oral ginger efficacy against postoperative nausea and vomiting: a randomized, double- blinded clinical trial. Iran Red Crescent Med J. 2013 Dec;15(12):e12268.

13. Shirvani MA, Motahari-Tabari N,Alipour A.The effect of mefenamic acid and ginger on pain relief in primary dysmenorrhea: a randomized clinical trial. Arch Gynecol Obstet. 2015 Jun;291(6):1277-81.

14. Rahnama P, Montazeri A, Huseini HF, Kianbakht S, Naseri M. Effect of Zingiber officinale R. rhizomes (ginger) on pain relief in primary dysmenorrhea: a placebo randomized trial. BMC Complement Altern Med. 2012 Jul;12:92.

15. Ozgoli G, Goli M, Moattar F. Comparison of effects of ginger, mefenamic acid, and ibuprofen on pain in women with primary dysmenorrhea. J Altern Complement Med. 2009 Feb;15(2):129-32.

16. Bartels EM, Folmer VN, Bliddal H, Altman RD, Juhl C, Tarp S, et al. Efficacy and safety of ginger in osteoarthritis patients: a meta-analysis of randomized placebo-controlled trials. Osteoarthritis Cartilage. 2015 Jan;23(1):13-21.

17. Al-Nahain A, Jahan R, Rahmatullah M. Zingiber officinale: a poten- 
tial plant against rheumatoid arthritis. Arthritis. 2014;2014:159089. https://doi.org/10.1155/2014/159089.

18. Baugh RF, Archer SM, Mitchell RB, Rosenfeld RM, Amin R, Burns $\mathrm{JJ}$, et al. Clinical practice guideline: tonsillectomy in children. Otolaryngol Head Neck Surg. 2011 Jan;144(1 Suppl):S1-30.

19. Sutters KA, Isaacson G. Posttonsillectomy pain in children. Am J Nurs. 2014 Feb;114(2):36-42.

20. Baliga MS, Haniadka R, Pereira MM, D'Souza JJ, Pallaty PL, Bhat $\mathrm{HP}$, et al. Update on the chemopreventive effects of ginger and its phytochemicals. Crit Rev Food Sci Nutr. 2011 Jul;51(6):499-523.

21. Apariman S, Ratchanon S, Wiriyasirivej B. Effectiveness of ginger for prevention of nausea and vomiting after gynecological laparoscopy. J Med Assoc Thai. 2006 Dec;89(12):2003-9.

22. Jiang X, Williams KM, Liauw WS, Ammit AJ, Roufogalis BD, Duke $\mathrm{CC}$, et al. Effect of ginkgo and ginger on the pharmacokinetics and pharmacodynamics of warfarin in healthy subjects. Br J Clin Pharmacol. 2005 Apr;59(4):425-32.

23. Lumb AB. Effect of dried ginger on human platelet function. Thromb Haemost. 1994 Jan;71(1):110-1.
24. Kashefi F, Khajehei M, Alavinia M, Golmakani E, Asili J. Effect of ginger (Zingiber officinale) on heavy menstrual bleeding: a placebocontrolled, randomized clinical trial. Phytother Res. 2015 Jan;29(1): 114-9.

25. Rayati F, Hajmanouchehri F, Najafi E. Comparison of anti-inflammatory and analgesic effects of ginger powder and Ibuprofen in postsurgical pain model: a randomized, double-blind, case-control clinical trial. Dent Res J (Isfahan). 2017 Jan-Feb;14(1):1-7.

26. Haghighi M, Khalvat A, Toliat T, Jallaei S. Comparing the effects of ginger (Zingiber officinale) extract and Ibuprofen on patients with osteoarthritis. Arch Iran Med. 2005 Oct;8(4):267-71.

27. Blumenthal M, Busse WR, Goldberg A, Gruenwald J, Hall T, Riggins $\mathrm{CW}$, et al. The complete German commission E monographs: therapeutic guide to herbal medicines. Austin: American Botanical Council; 1998.

28. Tiran D. Ginger to reduce nausea and vomiting during pregnancy: evidence of effectiveness is not the same as proof of safety. ComplementTher Clin Pract. 2012 Feb;18(1):22-5. 